\title{
A New Theory of the Structure of Matter
}

\author{
Ashot Agababyan* \\ * Chemistry Department, Erevan State University, Republic of Armenia. \\ * Head of Chemistry Laboratory "Scientific - Research Institute". Yerevan, Armenia.
}

Abstract: Mr. ASHOT MIKHAYELOVICH AGABABYAN innovated a New Theory of Structure of Matter in Chemistry Branch. He negated the existing, old theory and showed about its imperfection. Because

- The old theory wasn't able to calculate, i.e. it didn't give a mathematic proof.

- It explained through obstruction. It was stated the covalent (polar) chemical connection is formed by clash of the electron clouds.

- It wasn't able to predict. And this type of theory is considering as a matter of faith. But then it's not a scientific statement anymore.

Mr. ASHOT AGABABYAN represents a New Theory of the Structure of Matter.

- His theoretical calculation $100 \%$ matches with the experimental results.

- The new theory allows calculating with the accuracy up to the fifth digit after a comma.

- Predicts the length of the link. It has gotten a new possibility to count the inter-nucleus distance and the radius of atoms.

The above represented calculations were among the first ones. In case chemistry - colleagues confirm the test results and if magazine's editors print in their magazines, then we may send them the subsequent ones.

Keywords: Atom, Inter-nucleus Distance, Stereo-metrical Chemistry, Structure of Matter.

\section{Introduction}

Dr. ASHOT AGABABYAN, who is innovator - scientist, professor, is also an Honorable Chairman of the "ARMENIAN LEAGUE OF CULTURE" Non- Governmental Organization (2003), which is doing cultural and educational projects. He has published out this new theory in His monograph "Logic and Chemistry" in Yerevan. The main aim was to create a New Theory based on the inter-nuclear and the interatomic distance. The theory should be facilitate the calculation of inter-nuclear distances.

This is recommended for the publication department of chemistry and methods of teaching Kh.Abovyan Armenian State Pedagogical University. The editor was PhD S.Hovakimyan.

Magazine "Kantekh"- (collection of scientific papers) published Mr. AGABABYAN'S article "The logic in the teaching of chemistry" in 2010. The book is intended for professors and teachers of Chemistry. Here is shown the necessity of communication between the Logic Science and Chemistry. According to the rules of formal Logic Science here are the classical definitions of the basic concepts of Chemistry.

\section{Is The Theoretical Chemistry In A Crisis?}

\section{The Crisis Of The Theoretical Chemistry}

Yes, and indeed it's in the deep systematic crisis.

\section{Is That Evident That The Theoretical Chemistry Is In The Crisis?}

Yes, we have interviewed our colleagues - scientists, teachers and other specialists - with a question: when this theory was innovated and who is its author. There was no a single clear answer. Is that because of the long time-span, or of its anonymous and unclear nature?

\section{How The Crisis Is Confirmed?}

In the manifestations of the Theory.

- Contradictions

- The conformity to natural laws is not found

- It doesn't predict.

\section{What's The Cause of the Crisis?}

Stereochemistry is not an exact science. Stereochemistry should become stereo-metrical chemistry that is be able to calculate, not just measure it. We need a New Theory of the Structure of Matter that could be able to calculate, i.e. mathematically prove it and predict the length of the bond in compounds. There is a statement 
that Chemistry is an Exact Science. "We can't calculate", - says an academician Y.B.Zeldovich. "Each science consists of science as much, as much it consists of mathematics", used to say Leonardo Da Vinci.

\section{What To Do? Is There A Need For The New Theory For The Structure Of Matter?}

Yes. This kind of theory should be created. Conformity to natural laws should be found; identification of one, two or even three numbers is not conformity yet, then it would enable to predict. In case if opponents object that it's not possible, we may answer with A. Blok's words: "Even the impossible is possible". The opponents may argue: even if you are right, still, you are just criticising, but not suggesting anything new in return. We have an answer on this - There Are Suggestions!

6. What's The Potential Of The New Theory?

\begin{tabular}{|c|c|}
\hline The Old Theory & The New Theory \\
\hline \hline $\begin{array}{c}\text { Not able to calculate } \\
\text { Explains through obstruction } \\
\text { Noble to predict }\end{array}$ & $\begin{array}{l}\text { Calculate with accuracy } \\
\text { Explains through an angle } \\
\text { Predicts the length of the link }\end{array}$ \\
\hline
\end{tabular}

\section{Hydrogen}

\section{Is There Experimental Data On The Hydrogen Atom?}

According to the data given in the standard handbook, the radius of the hydrogen atom is equal to $\mathbf{0 , 0 5 3} \mathbf{n m}$. but in the molecule of hydrogen length of the link should be equal to $\mathbf{0 , 1 0 6 n m}$ accordingly.

\section{Are There Statistics On A Molecule Of Hydrogen?}

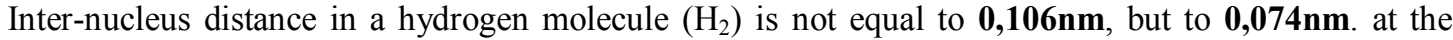
expense of the electron clouds. Check it in any chemistry text-book, and you'll learn that the radius is reducing up to $\mathbf{0 , 0 3 7} \mathbf{n m}$. which means that each hydrogen atom takes a half.

\section{How The Theory Explain These Discrepancies?}

The theory explains these experimental results as a clash of the electron clouds and clarifies by the following figure:

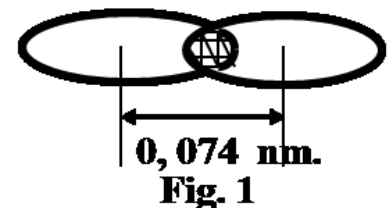

(The marked part is a place of the clash of the electron clouds)

\section{How About Carbon?}

To prove the antithesis, which states that there is no clash, we shall consider another element - Carbon (C), radius of which is equal to $\mathbf{0 , 0 7 7} \mathbf{~ n m}$. with the inter-nucleus distance (C-C) of $\mathbf{0 , 1 5 4 n m}$. (in diamond). There are no clashes, but there is a link, and a very strong one. Since diamond is the hardest mineral, there is no protection, but the link does exist. Here a contradiction appears, however the Theory doesn't explain it.

\section{What Is Not Right?}

The rule should be one - either covalent non-polar connection derives from the clash of the clouds (as it is in the case of a hydrogen molecule), or there is no clash at all, as it is with Carbon. "Ad hos", as philosophers say, is created especially for this kind of a case.

\section{How Do They Prove?}

It is stated the covalent (polar) chemical connection is formed by clash of the electron clouds. This statement can be accepted with no objection as a truth at its last stage: without any proofs and counting, i.e. as a matter of faith. But then it's not a scientific statement anymore.

The ancient sages had a saying: "It's not proved, it's not a truth". 
III. $\mathbf{H}-\mathbf{H}$

\section{What Are The Ways Of Explanation?}

The reduction of the length should be explained anyhow. Either through the clash, or without, there cannot be a third way.

\section{What to Do?}

"Let us count", - used to say Dr. Gottfried Leibniz. The inter-nucleus distance of $\mathbf{0 , 0 7 4 n m}$. in hydrogen molecule is interpreted through the bond angel which is equal to a 90 degree.

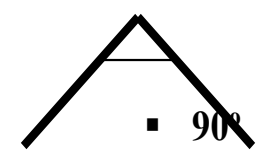

- $\mathrm{H} \quad \mathrm{H}$

Fig.2

15. How It Is Proved?

Take the theorem of Pythagoras and calculate.

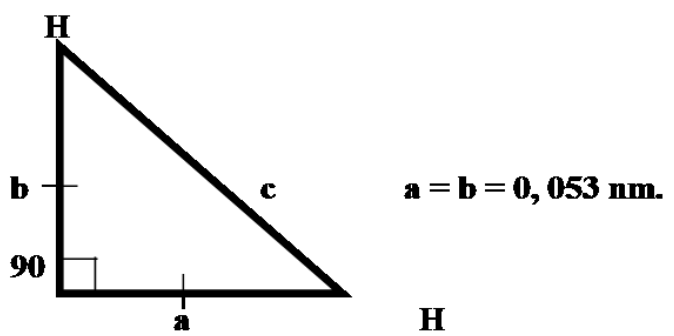

Fig-3

$\mathbf{c}^{2}=\mathbf{a}^{2}+\mathbf{b}^{2}$

$\mathrm{x}^{2}=\mathbf{0}, 053^{2}+\mathbf{0}, 053^{2}$

$x^{2}=0,002809+0,002809$

$\mathrm{x}^{2}=\mathbf{0}, 005618$

$\mathrm{x}=\sqrt{\mathbf{0 , 0 0 5 6 1 8}}=\mathbf{0 , 0 7 4}$

$\mathrm{x}=\mathbf{0 , 0 7 4 \mathrm { nm }}$

16. Did The Theoretical Calculation Match With The Experiment?

Yes, the theoretical calculation matched with the experimental results.

17. What Is The Degree Of Mistake?

A mistake up to 5 percent is considered permitted. However, in our case we have a complete matching.

18. Is It A Right Accuracy?

The accuracy is astonishing. It's applied to the seventh digit after the comma $-\mathbf{0 , 0 0 7 4 9 5 3 3} \mathbf{~ n m}$. And

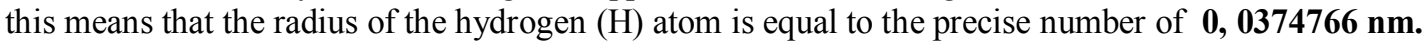

\section{What Is The Conclusion?}

\section{Conclusion}

To interpret the structure of the Hydrogen molecule not by the clash, but through the angle between them. While solving this task we, obviously, did not consider all the degrees from 1 to $360^{\circ}$ and did not counted up until they would match with the experimental results. It would have become an enormously long and difficult way. Here we have a small "know-how" which is due to the large number of calculations, would leave scope of this article, therefore will be described in subsequent articles. 


\section{What Is Then?}

According to the following plan: Hydrogen, Carbon, Oxygen, Nitrogen are in the system of 4 "Giants".

And then the other elements:

C-H, C-O, C-N, H-N, H-O, N-O.

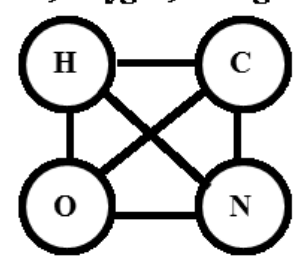

Fig-4

\section{Why Such A Sequence?}

The sequence has its significance.

Hydrogen is the simplest element. Here functions the principle from simple to complex.

Carbon is the main element of the organic chemistry. It is more that 10 million combinations, which consists 95 percent of the all known mattery.

Oxygen is a part of hydro-oxy-derivative from hydrocarbon.

Nitrogen, as the last one out from the 'giants' of the organic chemistry, is a consisting part of the amino-acids and albumen.

Only after considering the structure of these four 'giants' and their combinations, it would be possible to explain structure of any substance. All the remaining elements can be derived out from the combinations of these four elements with the other ones.

\section{What To Do With The Text - Books?}

If the interpretation of the structure of the hydrogen atom through the valent angle and not through the clash of the clouds is confirmed, then we need to re-write the books, including school text-books.

\section{What To Do With The Old Theory?}

It's already nine years since we turned into the $21^{\text {st }}$ century. In every 5 years the information is multiplied. The old theory, created in the middle of the previous century has become outdated and does not justify it. Hence, it would have been 10 times sent to the recycle bin of the history.

\section{What To Leave?}

Leave that what is true!!! Why do we still remember and use Mendeleev's Table and Law, Butlerov's theory, and Pythagoras's theorem? It is because they are true, isn't it?

\section{Regular Tetrahedron}

\section{What Is The Equal In The Tetrahedron?}

In the regular tetrahedron all the angles and sides are equal.

\section{What Parameters There Are In The Regular Tetrahedron?}

Height - (H), apothem - (h), the radius of inner lined of the globe - (r), the radius of out lined of the globe- (R), side - (a), surface - (S) and volume - (V).

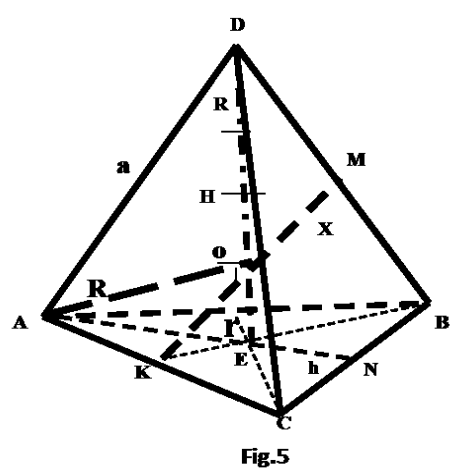

$$
\begin{aligned}
& \mathbf{H}=\mathbf{D E} \\
& \mathbf{a}=\mathbf{A D}=\mathbf{D B}=\mathbf{A C} \\
& \mathbf{R}=\mathbf{D O}=\mathbf{O A} \\
& \mathbf{r}=\mathbf{O E} \\
& \mathbf{h}=\mathbf{A N} \\
& \mathbf{X}=\mathbf{K M}
\end{aligned}
$$


27. How Does Relate The Radiuses With The Height Of The Tetrahedron?

$$
\underline{\mathbf{H}}=\mathbf{R}+\mathbf{r}
$$

D

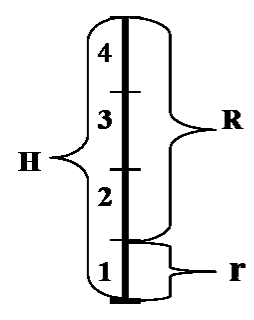

$\mathbf{E}$

Fig.6
$\mathbf{D}$

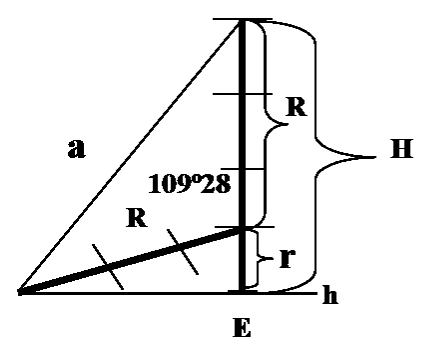

Fig.7

28. How Many Parts Are There In Of The H?

There are four parts in of the $\mathrm{H}$.

29. How Many Parts Are There In Of The R?

There are three parts in of the $\mathrm{R}$.

30. How Many Parts Are There In Of The R?

There is one part in the $\mathrm{r}$.

\section{Mathematics}

31. What Is The Radius (R) Of Circumscribed Sphere In The Tetrahedron?

$$
R=\frac{a \sqrt{6}}{4}
$$

32. What Is The Measure (A) Of The Side Of The Tetrahedron?

$$
a=\frac{4 R}{\sqrt{6}}
$$

33. What Is The Radius Of The Inscribed Sphere To The Tetrahedron?

$$
r=\frac{a \sqrt{6}}{12}
$$

34. What Is The Height (R) Of The Tetrahedron?

$$
H=\frac{a \sqrt{6}}{3}
$$

35. What Is The Apothem (H) Of The Tetrahedron?

$$
h^{2}=a^{2}-\left(\frac{a}{2}\right)^{2} \quad 4 h^{2}=3 a^{2} \quad h=\frac{a \sqrt{3}}{2}
$$

36. What Is The Distance (X) Between The Ribs Of The Tetrahedron?

$$
\mathbf{X}^{2}=\mathbf{R}^{2}-\left(\frac{\mathbf{a}}{2}\right)^{2} \quad \mathbf{X}=\sqrt{\mathbf{R}^{2}-\left(\frac{\mathbf{a}}{2}\right)^{2}}
$$




\section{Carbon}

37. Does The Principle Function For Other Elements?

The principle stating that the reduction of the combinations length between atoms happens due to the bond angel and not due to the clash of the electron clouds can be proved on the example of another element. Let's consider two allotrope modifications of Carbon - diamond and graphite.

\section{What Are The Statistics On Carbon?}

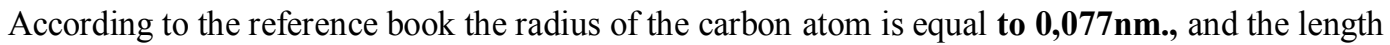
of the singular link in diamond is equal to $\mathbf{0 , 1 5 4} \mathbf{~ n m}$. (twice longer).

\section{What's The Angel In A Tetrahedron?}

The angle is equal to 109 degree and 28 minutes, which stands for the correct tetrahedron.

\section{What's The Dependence Between Parameters?}

In mathematics (geometry) as exact sciences, there is an established strict dependence between the edge and other parameters - the radius height (h) the radius of inscribed sphere (r) and the radius of circumscribed sphere (R), the surface (S) and the volume of tetrahedron.

\section{How To Check?}

Let's calculate. FORMULAS

$$
\begin{aligned}
& R=\frac{a \sqrt{6}}{4}=\frac{0,154 \times 2,45}{4}=0,09 \\
& a=\frac{4 R}{\sqrt{6}}=\frac{4 \times 0,077}{2,45}=0,12
\end{aligned}
$$

\section{Where Is The Mistake?}

If the edge is equal to $0,154 \mathbf{n m}$., then the $R$ is equal to $0,09 \mathbf{n m}$. But it should be equal $0,077 \mathbf{n m}$. If $\mathbf{R}=\mathbf{0 , 0 7 7} \mathbf{n m}$., then the edge is equal to $\mathbf{0}, \mathbf{1 2 n m}$., although it should be $=\mathbf{0}, \mathbf{1 5 4 \mathbf { n m }}$. So, it should be concluded, that one of the calculations is not correct. Either $\mathbf{R}$ of the carbon atom is not equal to $\mathbf{0 , 0 7 7} \mathbf{n m}$, or the inter-nucleus distance between the atoms is not equal to $\mathbf{0}, \mathbf{1 5 4 n m}$.

How this contradiction has been skipping out of our notice during 50 years? It's a paradox! You had to just simply take out the geometrical formulas to make this calculation.

\section{C- DIAMOND}

\section{Is There Connection Between The Edge And The Radius?}

Calculations reveal that there is no connection in the correct tetrahedron.

There is no link between the edge and the radius of the circumscribed sphere.

\section{Which Data Is Not Correct: The Experimental Or The Theoretical One?}

According to the logic, if in the theory does not fit with the results of the experiment, then either the theory is wrong, or the results. There cannot be another conclusion.

Experiments have been conducted thousand of times, and there cannot be errors, so the theory can nor comprehensibly explain the experimental results.

Let's take the diamond structure as the simplest one, has only singular links which are connected to the same kind of correct tetrahedrons.

For the simple example let us take two of them and develop for the better visualize. We get two tetrahedrons connected by the tops. 


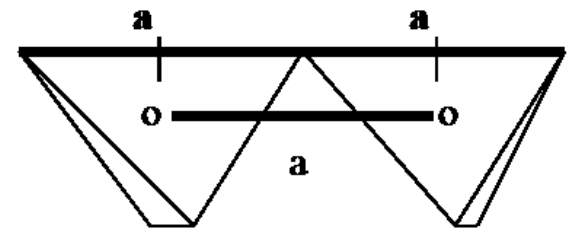

Fig.8

45. How To Prove?

It is easy to prove that the inter-nucleus distance in diamond (C-C) is equal to $\mathbf{0 . 1 5 4 n m}$.

a

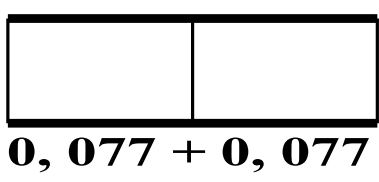

Fig.9

46. Where Is The Mistake?

The mistake originates from the inter-nucleus distance: $\mathbf{0 , 1 5 4}$ were divided into two, with the result of $\mathbf{0 . 0 7 7} \mathbf{n m}$. - a half to the each atom. Here again we get the same mistake as it was with the hydrogen atom. But even here we have got an angle. Since the mistake is revealed, we can start our calculations.

\section{What Is The Edge Equal To?}

The edge is equal to $\mathbf{0 , 1 5 4 n m}$. Then the radius cannot be equal to $\mathbf{0 , 0 7 7} \mathbf{n m}$.

\section{What's The Size Of The Edge?}

You may count. Let's we find the radius when $\mathbf{a}=\mathbf{0 , 1 5 4 n m}$.

\section{C- Diamond Calculations}

49. What Is The Measure Of The Radius?

$$
R=\frac{a \sqrt{6}}{4}
$$

50. Is It Measurable?

Of course.

$$
R=\frac{0,154 \quad 2,45}{4}=0,09 \mathrm{~nm} .
$$

Here is the exact measure of the radius, it is $\mathbf{0 , 0 9} \mathbf{~ n m}$.

\section{May We Count It Without Formula?}

Yes, of course we may calculation without formula; all the same we receive same result.

\section{What Did Give Us The Theory?}

For the second time theory proofed comes to correct the experiments results. And it is no coincidence, as some opponents might say, but it is conformity with the law.

The explanation of the longitude of the connections is given by valence angles. And this is found the exact radius of the atom. 
D

A

C

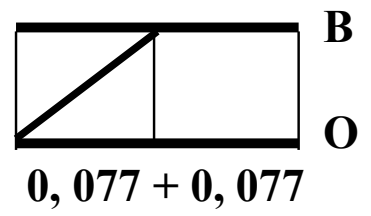

Fig.10

$$
\mathrm{AD}=\mathrm{DB}=\mathbf{0 , 0 7 7} \mathbf{\mathrm { nm } .} \quad \mathrm{AC}=\mathrm{BO}
$$

53. The Radius Of CD Equal To Half Of The Side AD?

No. $\mathbf{C D} \neq \mathbf{A D}$

\section{WHY?}

The circumscribed sphere in regular tetrahedron is never equal to the half of side, it always large. It means $\mathbf{C D}$ is large than AD.

So the radius is equal $0.09 \mathbf{~ n m}$. and the half of the side is $\mathbf{0 . 0 7 7} \mathbf{n m}$. All the rest parameters the regular tetrahedron the height, radius of inscribed sphere and etc., can be count as it above mentioned formula.

$$
\begin{aligned}
& H=0,154 \times 2,45 / 3=0,120 \mathrm{~nm} \\
& r=0,154 \times 2,45 / 12=0,03 \mathrm{~nm}
\end{aligned}
$$

\section{C-Graphite}

55. What Is The Distance Between The Layers Of Graphite?

The distance of the layers of graphite is $\mathbf{0 , 3 3 5} \mathbf{n m}$.

\section{How Does They Explain It?}

The old theory that explains the structure of the graphite, gives the following figure.

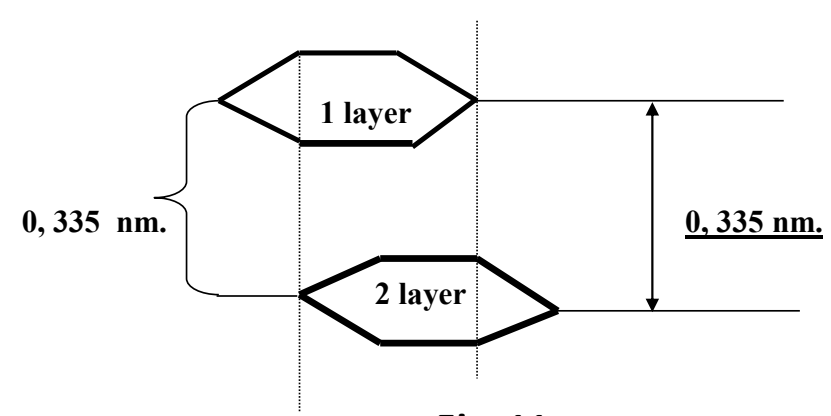

Fig .11

\section{What Are In The Between Of Strips?}

What is there instead of dotted line, theory, of course, is modestly silent, as well as with communication in a molecule of hydrogen and carbon.

\section{Is It Mean Not Theory, But It Is Hypothesis?}

Yes, because the theory is system of proofed hypothesis.

So, the logic is defected here, the theory is self - contradictory. According to the second law of the Logic Science the theory should not be internally inconsistent. Academician Frolov writes. "Our demand is that in the theory should not be logically contradictions". But what we see here. Most importantly mathemically they don't substantiate or confirm the calculations. Therefore, this is not a theory, but a hypothesis, an assumption.

Now we need a New Theory of the structure of matter in Chemistry, which could count, means prove it mathematically and could predict the length of the bond in compounds. 
59. And How To Make The Hypothesis To Theory? To count.

60. May To Count The Distance Between Of Strips? Let's count. See Fig 12.

\section{C - Graphite Calculation}

\section{From What To Begin?}

Having received exactly givens about the diamond, we can to count the graphite.

62. How?

Let's count the interval of the layers in the graphite. See Fig 12.

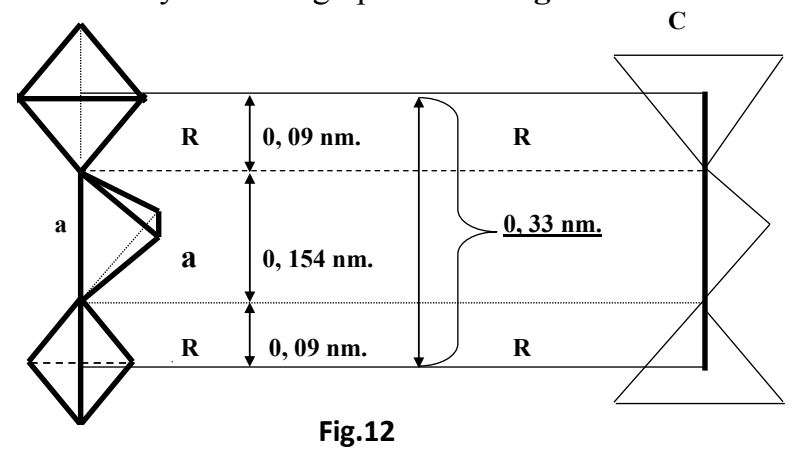

63. Is There Another Variant?

There is. See Fig 13.

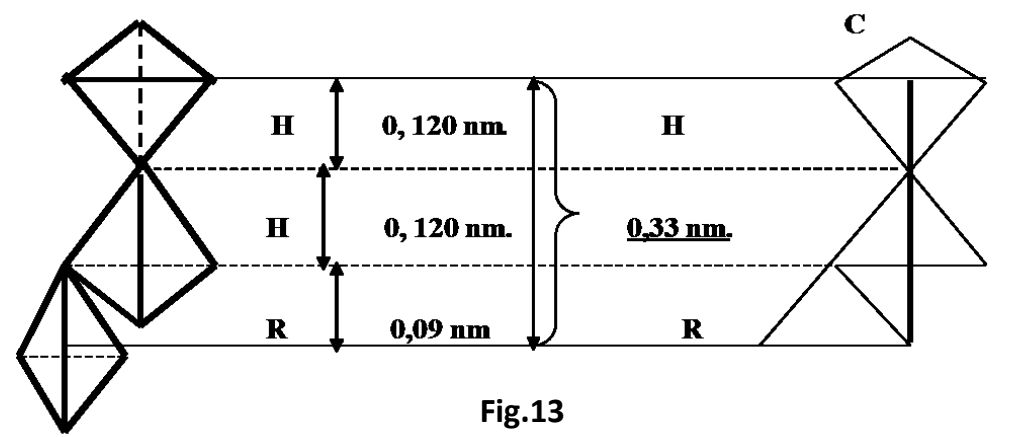

64. What Is The Equal Interval The Between Of The Layers In The Graphite?

$\mathrm{R}+\mathrm{a}+\mathrm{R}=\mathbf{0 , 0 9} \mathrm{nm} .+0,154 \mathrm{~nm} .+0,09 \mathrm{~nm} .=0,335 \mathrm{~nm}$.

65. What Is The Equal Interval In The Second Type?

$$
\mathrm{H}+\mathrm{R}+\mathrm{H}=\mathbf{0 , 1 2 0} \mathrm{nm} .+\mathbf{0 , 0 9} \mathrm{nm} .+\mathbf{0}, 120 \mathrm{~nm} .=0,335 \mathrm{~nm} .
$$

66. Are They Equal To Each Other?

Yes. $\mathbf{2 H}+\mathbf{R}=\mathbf{2} \mathbf{R}+\mathbf{a}=\mathbf{0}, \mathbf{3 3 5} \mathbf{n m}$. 


\section{Results And Conclusion}

67. Does The Theory Correspond With The Practise?

Third time the counts of theory correspond with practical $100 \%$.

It means is found the regularity.

\begin{tabular}{|c|c|c|}
\hline Element & Experimental given & Theoretical given \\
\hline H & $0,0074 \mathbf{n m}$. & $\mathbf{0 , 0 0 7 4} \mathbf{~ n m}$. \\
\hline C & $\mathbf{a}=0,077 \mathbf{n m}$. & $\mathbf{a}=0,077 \mathrm{~nm} . \quad \mathbf{R}=\mathbf{0 , 0 9 \mathrm { nm }}$. \\
\hline
\end{tabular}

68. What We Have Done?

We considered the existing theory (hypothesis) of the Matter Structure and convinced about its imperfection. And now Importance of the New Theory is undoubtedly obvious.

\section{What Was Created?}

A New Theory of Matter Structure has established. The new theory allows calculating; therefore with the accuracy up to the fifth digit after a comma the Matter Structure can be established. We have got a new possibility to count the inter-nucleus distance and the radius of atoms. No doubt, that the theory should find its test approval on the remaining more than 100 elements.

The above represented calculations were among the first ones. In case our colleagues confirm the test results, we may send them the subsequent ones.

\section{What Should Be Corrected?}

In the text-books instead of dotted lines a correct tetrahedron should be drawn in between of the graphite layers - just one tetrahedron 'standing' on the edge, or its angular isomer 'hanging' from the side. There can be other variations, but that that should be a tetrahedron between the layers - is beyond any doubts.

\section{Do The Qualities Of The Substance Depend On The Signs?}

Yes, notwithstanding the statement that the signs are qualities. That means, that Butlerov was not correct when he stated that the qualities depend on the structure, and the structure is the signs. According to Butlerov's theory - "Qualities of matter depend on structure". And this is a firm statement.

\section{Why The Way To The Theory Was So Long?}

Decades were spent on calculations, in sake of get rid of contradictions.

"The way to the Truth is the One, but they are countless ones towards Delusion" - stated Sages.

\section{P.S. "The most significant discovery will be done in Chemistry" - D. M. Mendeleev.}

[1] B. V. Grigoriev, "Classical Logic", 1996.

\section{References}

[2] P. M. Zorky, "The fundamental concepts of chemistry", 1996. 